\title{
Low-Rank Channel and Interference Estimation in mm-Wave Massive Antenna Arrays
}

\author{
G. Soatti*, A. Murtada*, M. Nicoli*, J. Gambini ${ }^{\dagger}$, U. Spagnolini* \\ ${ }^{*}$ Politecnico di Milano, Milan, Italy \\ ${ }^{\dagger}$ Huawei Technologies, Milan Research Center, Milan, Italy \\ Email:\{gloria.soatti,monica.nicoli,umberto.spagnolini\}@polimi.it,ahmedabdelnaser.murtada@mail.polimi.it, \\ jonathan.gambini@huawei.com
}

\begin{abstract}
Millimeter wave (mm-Wave) communications are characterized by wideband channels with few directional paths, mostly in line-of-sight. Antenna arrays are mandatory to cope with severe path-loss, and the resulting channel response is sparse in the space-time (ST) domain. This paper addresses the sparsity by proposing a channel estimation method that exploits the algebraic structure of channel and interference, without requiring complex antenna-array calibration procedures. The method relies on the recognition that the ST channel is lowrank and exhibits slowly and fast-varying features (angles/delays of arrival and fading amplitudes, respectively) and, accordingly, that the interference has a slowly-varying spatial covariance with fast-varying amplitudes. The accuracy of the estimation of quasistationary components is increased by introducing averaging mechanisms over multiple sequences. Numerical results show that: i) rank-1 is an effective channel-interference representation in $\mathrm{mm}$-Wave setting with severe interference; ii) fundamental limits (derived in closed form) prove the remarkable performance gains in terms of signal-to interference ratio; iii) circular array arrangement with directive elements is preferable compared to square or triangular configurations.
\end{abstract}

Index Terms-mm-Wave, space-time channel estimation, subspace methods, antenna array.

\section{INTRODUCTION}

The ambitious throughput requirements envisioned for $5 \mathrm{G}$ applications have paved the way to wireless systems operating in the millimeter wave (mm-Wave) region [1]. The availability of larger bandwidths (say over $100 \mathrm{MHz}$ ), compared to sub$6 \mathrm{GHz}$ regions, comes at the price of increased attenuation and fading (e.g., due to rain). Distributed antenna systems with arrays of radiating elements are thus necessary to guarantee area coverage and also for efficient management of interuser interference [2,3]. Remote antenna units (RAUs) can be deployed either in massive multiple-input-multiple-output (MIMO) configuration [4], or with smaller number of antennas to limit the power consumption of mm-Wave hardware [5]. In both configurations, accurate knowledge of the channel response over the space-time (ST) domain (i.e., over antennas and delays) is key for designing precoding/decoding schemes that fully exploit the array gain by adapting the transmissionreception strategy to the dynamic multi-user environment using spatial multiplexing/diversity techniques [6,7].

Conventional ST channel estimation methods fail to optimize the overhead versus accuracy trade-off that arises in $\mathrm{mm}$-Wave systems, mostly when employing a large number of antennas and operating in critical signal-to-noise-ratio (SNR) before the beamforming gain [8]. Efficient techniques for sparse ST channels estimate a parsimonious set of parameters that fully describes the mm-Wave channel in terms of angles of arrival and departure, power gains and delays of few channel paths. Compressive sensing algorithms are tailored to estimate these few parameters, but require a calibrated array to preserve the manifold association. Training-based beamforming and combining vectors for hybrid analog/digital systems have been proposed for estimating the narrowband [9] and wideband [10] geometric parameters. On the other hand, subspace methods rely on the evaluation of the singular vectors of the MIMO channel [11]-[13]. These methods, however, do not adapt to the different SNR conditions that may affect the dynamic propagation environment, and/or do not take into account the interference pattern when estimating the ST channel features (or the corresponding beamforming).

In this paper, we focus on channel estimation for timeslotted single-input-multiple-output (SIMO) mm-Wave systems, as preliminary to multi-user MIMO, and we propose a subspace method that exploits the low-rank (LR) algebraic structure of both user and interferer channels, as well as the different degrees of variability of the related features. The algebraic structure arises from the combination of slowly (angles/delays) and fast-varying (fading amplitudes) channel components with the slowly varying spatial covariance of the interference. Instead of an explicit estimation of the geometric multipath parameters (sensitive to array calibration errors), we consider a non-parametric approach that searches for the most parsimonious ST subspace describing the SIMO channel [14].

Since the mm-Wave channel has a LR structure, the slowlyvarying features are obtained through the estimation of the dominant ST channel subspaces - comprehensive of the interference - from the observation of several consecutive slots, while the fast-varying Rice-faded amplitudes are tracked on a slot-by-slot basis. To assess the performance, we derive fundamental limits to the mean square error (MSE) of the channel estimate for unknown ST rank orders. With respect to [14], here the method is specifically tailored to mm-Wave systems with different antenna array configurations (circular, square and triangular), and the analytical performance is first derived here by taking into account the bias effect due to the unknown rank orders. Numerical results confirm that the 


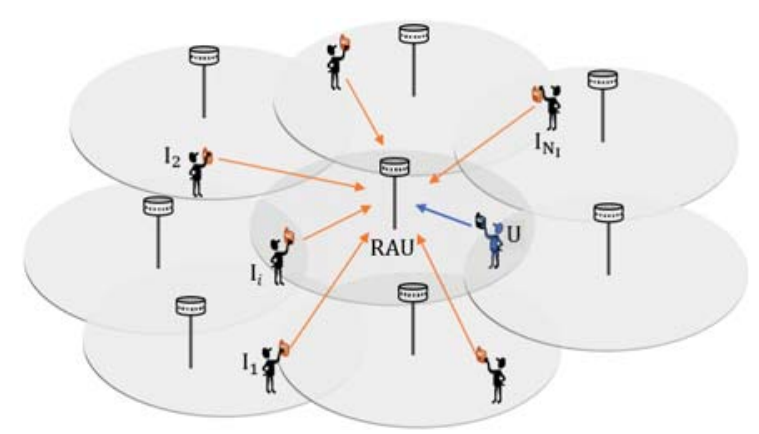

Figure 1. A mm-Wave scenario where a single user communicates with a RAU using multiple-antennas. Presence of $N_{I}$ interferers in the environment.

maximum likelihood estimate (MLE) subject to LR provides significant performance gains compared to the conventional full-rank (FR) one and that rank-1 is an effective channelinterference representation in mm-Wave settings with severe interference. Numerical validations for different array configurations show that the circular array is preferable when the antenna element is directive.

\section{Signal Model}

We consider a time-slotted wireless communication system where a single-antenna user (U) transmits data to a RAU equipped with $M$ antennas. The mm-Wave scenario includes also the presence of several interferers as shown in Fig. 1. Let $\mathbf{Y}(\ell) \in \mathbb{C}^{M \times N}$ be the matrix gathering the $N$ samples (at symbol rate) of the signal received within the $\ell$ th training slot ( $\ell=1, \ldots, L)$ by the $M$ antennas. The baseband equivalent signal model can be written as:

$$
\mathbf{Y}(\ell)=\mathbf{H}(\ell) \mathbf{X}+\mathbf{N}(\ell),
$$

where $\mathbf{H}(\ell) \in \mathbb{C}^{M \times W}$ is the block-fading frequency-selective SIMO channel with temporal support length $W$ within the $\ell$ th slot and $\mathbf{X} \in \mathbb{C}^{W \times N}$ is the convolution matrix of the channel with the training sequence of length $N$. Moreover, the co-channel interference and the background noise are modeled as an additive zero-mean Gaussian process $\mathbf{N}(\ell)=$ $[\mathbf{n}(1, \ell) \cdots \mathbf{n}(N, \ell)] \in \mathbb{C}^{M \times N}$, that is assumed to be temporally uncorrelated but spatially correlated (due to geometrical arrangement of interferers) with unknown spatial covariance Q, i.e., $\mathbb{E}\left[\mathbf{n}(n, \ell) \mathbf{n}^{\mathrm{H}}(n-k, \ell)\right]=\mathbf{Q} \delta(k)$, with $[\mathbf{Q}]_{m, m}=\sigma^{2}$ being the noise power at each antenna.

The sparse multipath structure of the ST mm-Wave channel is modeled as a combination of $P$ paths:

$$
\mathbf{H}(\ell)=\sum_{p=1}^{P} \alpha_{p}(\ell) \mathbf{a}\left(\theta_{p}\right) \mathbf{g}^{\mathrm{T}}\left(\tau_{p}\right)=\mathbf{A D}(\ell) \mathbf{G}^{\mathrm{T}},
$$

where $\alpha_{p}(\ell)$ is the slot-dependent fading amplitude of path $p$, while $\tau_{p}$ and $\theta_{p}$ are the related delay and angle of arrival that are typically invariant for a large number of slots and can be considered as slot-independent over the $L$ slots [14]. Moreover, $\mathbf{a}\left(\theta_{p}\right) \in \mathbb{C}^{M \times 1}$ denotes the response of the antenna array receiver, while the vector $\mathbf{g}\left(\tau_{p}\right) \in \mathbb{R}^{W \times 1}$ collects the samples of the delayed waveform $g(\tau)$ representing the convolution of the transmitted pulse with the matched filter at the receiver.
A compact form of the channel matrix is shown in the third term in (2), where the multipath parameters are gathered into spatial $\mathbf{A}=\left[\mathbf{a}\left(\theta_{1}\right) \cdots \mathbf{a}\left(\theta_{P}\right)\right]$, temporal $\mathbf{G}=\left[\mathbf{g}\left(\tau_{1}\right) \cdots \mathbf{g}\left(\tau_{P}\right)\right]$ and diagonal $\mathbf{D}(\ell)=\operatorname{diag}\left(\alpha_{1}(\ell) \cdots \alpha_{P}(\ell)\right)$ matrices.

In order to avoid a joint estimation of angles and delays, which is highly complex and sensitive to antenna calibration errors, we propose to model the ST channel (2) in terms of unstructured slowly/fast varying matrices, by following the approach in [14]. Let $r_{\mathrm{S}}=\operatorname{rank}[\mathbf{A}] \leq \min (P, M)$ and $r_{\mathrm{T}}=\operatorname{rank}[\mathbf{G}] \leq \min (P, W)$ be respectively the spatial and temporal diversity orders or, equivalently, the numbers of resolvable angles and delays (given the array aperture and the signal bandwidth). Using the above LR constraints, we rewrite the channel (2) as the combination of three full-rank matrices:

$$
\mathbf{H}(\ell)=\mathbf{U}_{\mathrm{S}} \boldsymbol{\Gamma}(\ell) \mathbf{U}_{\mathrm{T}}^{\mathrm{H}},
$$

where $\boldsymbol{\Gamma}(\ell)\left(r_{\mathrm{S}} \times r_{\mathrm{T}}\right)$ is a fast-fading (slot-dependent) matrix, while the spatial $\mathbf{U}_{\mathrm{S}}\left(M \times r_{\mathrm{S}}\right)$ and temporal $\mathbf{U}_{\mathrm{T}}\left(W \times r_{\mathrm{T}}\right)$ slow-fading (slot-independent) matrices collect the eigenvectors of the spatial and temporal correlations for the channel matrix $\mathbf{H}(\ell)$, respectively defined as $\mathbf{R}_{\mathrm{S}}=\mathrm{E}\left[\mathbf{H}(\ell) \mathbf{H}^{\mathrm{H}}(\ell)\right]$ and $\mathbf{R}_{\mathrm{T}}=\mathrm{E}\left[\mathbf{H}^{\mathrm{H}}(\ell) \mathbf{H}(\ell)\right]$.

\section{LOW-RAnK ChANNEL Estimation}

In this section, we present the LR method for the MLE of the channel matrices $\{\mathbf{H}(\ell)\}_{\ell=1}^{L}$ and the noise covariance $\mathbf{Q}$ from the received signals $\{\mathbf{Y}(\ell)\}_{\ell=1}^{L}$, under the LR constraint (3) and for known rank orders $\left(r_{\mathrm{S}}, r_{\mathrm{T}}\right)$ (Sec. III-A). We then derive a closed form expression for the asymptotic performance of the LR method for unknown rank orders, using as values $\hat{r}_{\mathrm{S}}, \hat{r}_{\mathrm{T}}$ for the LR estimation (Sec. III-B).

\section{A. Low-Rank MLE Method}

The conventional FR (or unconstrained) MLE of the channel $\mathbf{H}(\ell)$ and the noise covariance matrix $\mathbf{Q}$ is given as:

$$
\begin{aligned}
& \hat{\mathbf{H}}_{\mathrm{u}}(\ell)=\mathbf{R}_{y x}(\ell) \mathbf{R}_{x x}^{-1}, \\
& \hat{\mathbf{Q}}_{\mathrm{u}}=\frac{1}{N L} \sum_{\ell=1}^{L}\left(\mathbf{R}_{y y}(\ell)-\mathbf{R}_{y x}(\ell) \mathbf{R}_{x x}^{-1} \mathbf{R}_{x y}(\ell)\right),
\end{aligned}
$$

where $\mathbf{R}_{y x}(\ell)=\mathbf{Y}(\ell) \mathbf{X}^{\mathrm{H}}, \mathbf{R}_{x x}=\mathbf{X} \mathbf{X}^{\mathrm{H}}$ and $\mathbf{R}_{y y}(\ell)=$ $\mathbf{Y}(\ell) \mathbf{Y}^{\mathrm{H}}(\ell)$ are the sample correlation matrices $\left(\mathbf{R}_{x x}\right.$ is assumed to be positive definite).

The LR MLE under the constraint (3), for rank orders $\left(r_{\mathrm{S}}, r_{\mathrm{T}}\right)$, is obtained as a post-processing of the FR MLE as:

$$
\hat{\mathbf{H}}_{\mathrm{LR}}(\ell)=\hat{\mathbf{Q}}_{\mathrm{u}}^{\frac{\mathrm{H}}{2}} \hat{\tilde{\mathbf{H}}}_{\mathrm{LR}}(\ell) \mathbf{R}_{x x^{-\frac{\mathrm{H}}{2}}}
$$

where $\hat{\tilde{\mathbf{H}}}_{\mathrm{LR}}(\ell)=\hat{\tilde{\mathbf{P}}}_{\mathrm{S}, r_{\mathrm{S}}} \tilde{\mathbf{H}}_{\mathrm{u}}(\ell) \hat{\tilde{\mathbf{P}}}_{\mathrm{T}, r_{\mathrm{T}}}$ denotes the whitened LR channel estimate and $\tilde{\mathbf{H}}_{\mathrm{u}}(\ell)$ the whitened FR one

$$
\tilde{\mathbf{H}}_{\mathrm{u}}(\ell)=\hat{\mathbf{Q}}_{\mathrm{u}}^{-\frac{\mathrm{H}}{2}} \hat{\mathbf{H}}_{\mathrm{u}}(\ell) \mathbf{R}_{x x}^{\frac{\mathrm{H}}{2}} \text {. }
$$

Moreover, $\hat{\tilde{\mathbf{P}}}_{\mathrm{S}, r_{\mathrm{S}}}=\hat{\tilde{\mathbf{U}}}_{\mathrm{S}, r_{\mathrm{S}}} \hat{\tilde{\mathbf{U}}}_{\mathrm{S}, r_{\mathrm{S}}}^{\mathrm{H}}$ is the projector onto the subspace spanned by the $r_{\mathrm{S}}$ leading eigenvectors $\hat{\tilde{\mathbf{U}}}_{\mathrm{S}, r_{\mathrm{S}}}$ of the spatial correlation matrix $\hat{\tilde{\mathbf{R}}}_{\mathrm{S}}(L)$. Similarly, $\hat{\tilde{\mathbf{P}}}_{\mathrm{T}, r_{\mathrm{T}}}=$ $\hat{\tilde{\mathbf{U}}}_{\mathrm{T}, r_{\mathrm{T}}} \hat{\tilde{\mathbf{U}}}_{\mathrm{T}, r_{\mathrm{T}}}^{\mathrm{H}}$ is the projector onto the subspace spanned by 
the $r_{\mathrm{T}}$ leading eigenvectors $\hat{\tilde{\mathbf{U}}}_{\mathrm{T}, r_{\mathrm{T}}}$ of the temporal correlation matrix $\hat{\tilde{\mathbf{R}}}_{\mathrm{T}}(L)$. The two correlation matrices are given by:

$$
\begin{aligned}
\hat{\tilde{\mathbf{R}}}_{\mathrm{S}}(L) & =\frac{1}{L} \sum_{\ell=1}^{L} \tilde{\mathbf{H}}_{\mathrm{u}}(\ell) \tilde{\mathbf{H}}_{\mathrm{u}}^{\mathrm{H}}(\ell), \\
\hat{\tilde{\mathbf{R}}}_{\mathrm{T}}(L) & =\frac{1}{L} \sum_{\ell=1}^{L} \tilde{\mathbf{H}}_{\mathrm{u}}^{\mathrm{H}}(\ell) \tilde{\mathbf{H}}_{\mathrm{u}}(\ell) .
\end{aligned}
$$

Since the spatial and temporal bases are estimated by averaging the signals over $L$ time slots, the accuracy is expected to increase with $L$ when the fading is uncorrelated. Notice that the LR method (6) incorporates the interference covariance into the ST channel estimate, thus taking into account possible directional interference when computing $\hat{\tilde{\mathbf{H}}}_{\mathrm{LR}}(\ell)$, with $\hat{\tilde{\mathbf{P}}}_{\mathrm{S}, r_{\mathrm{S}}}$ and $\hat{\tilde{\mathbf{P}}}_{\mathrm{T}, r_{\mathrm{T}}}$ acting as spatial and temporal filters.

The LR estimate of the noise covariance matrix

$$
\hat{\mathbf{Q}}(L)=\frac{1}{N L} \sum_{\ell=1}^{L} \hat{\mathbf{N}}(\ell) \hat{\mathbf{N}}(\ell)^{\mathrm{H}},
$$

is obtained from the residuals of the channel estimation $\hat{\mathbf{N}}(\ell)=\mathbf{Y}(\ell)-\hat{\mathbf{H}}(\ell) \mathbf{X}$.

\section{B. Performance Analysis for Unknown Rank Orders}

A closed form expression for the MSE of the LR channel estimate is derived here as $\operatorname{MSE}_{\mathrm{LR}}=\mathbb{E}\left[\|\triangle \mathbf{H}(\ell)\|^{2}\right]$, with $\triangle \mathbf{H}(\ell)=\hat{\mathbf{H}}_{\mathrm{LR}}(\ell)-\mathbf{H}(\ell)$ and where $\|\mathbf{A}\|^{2}=\operatorname{tr}\left[\mathbf{A} \mathbf{A}^{\mathrm{H}}\right]$ denotes the Frobenius norm of matrix $\mathbf{A}$. The impact of a possible mismatch between the rank orders used in the estimation $\left(\hat{r}_{\mathrm{S}}, \hat{r}_{\mathrm{T}}\right)$ and the true channel rank orders $\left(r_{\mathrm{S}}, r_{\mathrm{T}}\right)$ is taken into account to comply with practical mm-Wave scenarios where the rank orders are unknown and need to be selected so as to minimize the MSE of the estimate. The choice $\hat{r}_{\mathrm{S}}=r_{\mathrm{S}}$ and $\hat{r}_{T}=r_{\mathrm{T}}$ is the minimum order that gives an unbiased channel estimate, but in general it does not provide the lowest MSE. For low signal-to-interferencenoise ratio (SINR), that is likely to occur in mm-Wave systems with transmit/receive misalignments or high path-loss, it might be more convenient to choose a biased estimator with lower rank (i.e., $\hat{r}_{\mathrm{S}}<r_{\mathrm{S}}$ or $\hat{r}_{T}<r_{\mathrm{T}}$ ) to trade distortion (due to under-parameterization) for estimate error. To highlight this, we derive the LR performance for any rank-order selection with $1 \leq \hat{r}_{\mathrm{S}} \leq M$ and $1 \leq \hat{r}_{T} \leq W$. The performance, here referred to as asymptotic MSE bound, is computed analytically assuming that the training sequence is very long (say $N \rightarrow \infty$ ) and the number of slots is $L \rightarrow \infty$. The asymptotic MSE bound will be used in the following sections to analyze the impact of rank-order selection on the performance of $\mathrm{mm}$ Wave systems. Note that for $N, L \rightarrow \infty$ the noise covariance $\mathbf{Q}$ can be considered as known (as $\hat{\mathbf{Q}}(L) \rightarrow \mathbf{Q}$ ) and the estimated ST projectors equal to the projectors onto the subspaces spanned by the whitened channel $\tilde{\mathbf{H}}(\ell)=\mathbf{Q}^{-\frac{\mathrm{H}}{2}} \mathbf{H}(\ell) \mathbf{R}_{x x}^{\frac{\mathrm{H}}{2}}$, i.e., $\tilde{\tilde{\mathbf{P}}}_{\mathrm{S}, r_{\mathrm{S}}} \rightarrow \tilde{\mathbf{P}}_{\mathrm{S}, r_{\mathrm{S}}}=\tilde{\mathbf{U}}_{\mathrm{S}} \tilde{\mathbf{U}}_{\mathrm{S}}^{\mathrm{H}}$ and $\hat{\tilde{\mathbf{P}}}_{\mathrm{T}, r_{\mathrm{T}}} \rightarrow \tilde{\mathbf{P}}_{\mathrm{T}, r_{\mathrm{T}}}=\tilde{\mathbf{U}}_{\mathrm{T}} \tilde{\mathbf{U}}_{\mathrm{T}}^{\mathrm{H}}$, with $\tilde{\mathbf{U}}_{\mathrm{S}}=\mathbf{Q}^{-\frac{\mathrm{H}}{2}} \mathbf{U}_{\mathrm{S}}$ and $\tilde{\mathbf{U}}_{\mathrm{T}}=\mathbf{R}_{x x}^{\frac{1}{2}} \mathbf{U}_{\mathrm{T}}$.

According to (6), the LR estimate error is $\triangle \mathbf{H}(\ell)=$ $\mathbf{Q}^{\frac{\mathrm{H}}{2}} \triangle \tilde{\mathbf{H}}(\ell) \mathbf{R}_{x x}^{-\frac{\mathrm{H}}{2}}$, where $\Delta \tilde{\mathbf{H}}(\ell)=\tilde{\hat{\mathbf{H}}}_{\mathrm{LR}}(\ell)-\tilde{\mathbf{H}}(\ell)$ denotes the LR estimate error in the whitened domain and the whitened multipath channel is $\tilde{\mathbf{H}}(\ell)=\sum_{p} \alpha_{p}(\ell) \tilde{\mathbf{a}}\left(\theta_{p}\right) \tilde{\mathbf{g}}^{\mathrm{T}}\left(\tau_{p}\right)$, with $\tilde{\mathbf{a}}=$
$\mathbf{Q}^{-\mathrm{H} / 2} \mathbf{a}$ and $\tilde{\mathbf{g}}=\left(\mathbf{R}_{x x}^{1 / 2} \mathbf{g}\right)^{*}$. From (1), (6) and (7), after some algebraic manipulations, it can be shown that the whitened LR error based on rank orders $\left(\hat{r}_{\mathrm{S}}, \hat{r}_{T}\right)$ is the sum of two terms, one accounting for the distortion due to rank mismatch (i.e., for $\hat{r}_{\mathrm{S}}<r_{\mathrm{S}}$ or $\hat{r}_{T}<r_{\mathrm{T}}$ ) and the other for the noise:

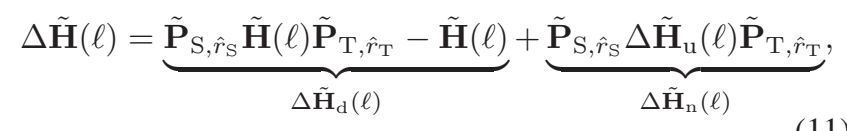

where $\Delta \tilde{\mathbf{H}}_{\mathrm{u}}(\ell)=\tilde{\mathbf{H}}_{\mathrm{u}}(\ell)-\tilde{\mathbf{H}}(\ell)$ is the whitened FR error. After some manipulation, the distortion term can be rewritten as $\Delta \tilde{\mathbf{H}}_{\mathrm{d}}(\ell)=\Delta \tilde{\mathbf{P}}_{\mathrm{S}} \tilde{\mathbf{H}}(\ell) \Delta \tilde{\mathbf{P}}_{\mathrm{T}}-\Delta \tilde{\mathbf{P}}_{\mathrm{S}} \tilde{\mathbf{H}}(\ell)-\tilde{\mathbf{H}}(\ell) \Delta \tilde{\mathbf{P}}_{\mathrm{T}}$, where $\Delta \tilde{\mathbf{P}}_{\mathrm{S}}=\tilde{\mathbf{P}}_{\mathrm{S}, r_{\mathrm{S}}}-\tilde{\mathbf{P}}_{\mathrm{S}, \hat{r}_{\mathrm{S}}}$ and $\Delta \tilde{\mathbf{P}}_{\mathrm{T}}=\tilde{\mathbf{P}}_{\mathrm{T}, r_{\mathrm{T}}}-\tilde{\mathbf{P}}_{\mathrm{T}, \hat{r}_{\mathrm{T}}}$ denote the differences between the true projectors $\left(\tilde{\mathbf{P}}_{\mathrm{S}, r_{\mathrm{S}}}, \tilde{\mathbf{P}}_{\mathrm{T}, r_{\mathrm{T}}}\right)$ and the projectors onto the spatial-temporal subspaces of the channel (3) selected using the dimensions $\hat{r}_{\mathrm{S}}$ and $\hat{r}_{\mathrm{T}}\left(\tilde{\mathbf{P}}_{\mathrm{S}, \hat{r}_{\mathrm{S}}}, \tilde{\mathbf{P}}_{\mathrm{T}, \hat{r}_{\mathrm{T}}}\right)$.

Since the two terms in (11) are uncorrelated, we get that the MSE is the sum of the two corresponding MSE terms:

$$
\operatorname{MSE}_{\mathrm{LR}}\left(\hat{r}_{\mathrm{S}}, \hat{r}_{\mathrm{T}}\right)=\operatorname{MSE}_{\mathrm{d}}\left(\hat{r}_{\mathrm{S}}, \hat{r}_{\mathrm{T}}\right)+\operatorname{MSE}_{\mathrm{n}}\left(\hat{r}_{\mathrm{S}}, \hat{r}_{\mathrm{T}}\right) \text {. }
$$

The first MSE term in (12) accounts for the distortion introduced by the rank reduction. It is zero for $\hat{r}_{\mathrm{S}} \geq r_{\mathrm{S}}$ and $\hat{r}_{\mathrm{T}} \geq r_{\mathrm{T}}$ [14], while for under-estimated ranks it is:

$$
\begin{aligned}
\operatorname{MSE}_{\mathrm{d}}\left(\hat{r}_{\mathrm{S}}, \hat{r}_{T}\right)=\sum_{p=1}^{P} & \sigma_{p}^{2}\left(\Phi\left(\Delta \tilde{\mathbf{P}}_{\mathrm{S}} \tilde{\mathbf{R}}_{\mathrm{S}_{p}}, \mathbf{Q}\right) \Phi\left(\Delta \tilde{\mathbf{P}}_{\mathrm{T}} \tilde{\mathbf{R}}_{\mathrm{T}_{p}}, \mathbf{R}_{x x}^{-1}\right)\right. \\
& -\Phi\left(\tilde{\mathbf{R}}_{\mathrm{S}_{p}}, \mathbf{Q}\right) \Phi\left(\Delta \tilde{\mathbf{P}}_{\mathrm{T}} \tilde{\mathbf{R}}_{\mathrm{T}_{p}}, \mathbf{R}_{x x}^{-1}\right) \\
& \left.-\Phi\left(\Delta \tilde{\mathbf{P}}_{\mathrm{S}} \tilde{\mathbf{R}}_{\mathrm{S}_{p}}, \mathbf{Q}\right) \Phi\left(\tilde{\mathbf{R}}_{\mathrm{T}_{p}}, \mathbf{R}_{x x}^{-1}\right)\right)
\end{aligned}
$$

where $\sigma_{p}^{2}=\mathbb{E}\left(\left|\alpha_{p}(\ell)\right|^{2}\right)$ is the power of the path $p, \tilde{\mathbf{R}}_{\mathrm{S}_{p}}=$ $\tilde{\mathbf{a}}\left(\theta_{p}\right) \tilde{\mathbf{a}}^{\mathrm{H}}\left(\theta_{p}\right)$ and $\tilde{\mathbf{R}}_{\mathrm{T}_{p}}=\tilde{\mathbf{g}}\left(\tau_{p}\right) \tilde{\mathbf{g}}^{\mathrm{T}}\left(\tau_{p}\right)$ are the spatial and temporal channel correlation matrices in the whitened domain and with $\Phi(\mathbf{P}, \mathbf{A})=\operatorname{tr}\left\{\mathbf{A}^{\mathrm{H} / 2} \mathbf{P} \mathbf{A}^{1 / 2}\right\}$. The second MSE term in (12) depends mainly on the interference:

$$
\operatorname{MSE}_{\mathrm{n}}\left(\hat{r}_{\mathrm{S}}, \hat{r}_{\mathrm{T}}\right)=\Phi\left(\tilde{\mathbf{P}}_{\mathrm{S}, \hat{r}_{\mathrm{S}}}, \mathbf{Q}\right) \Phi\left(\tilde{\mathbf{P}}_{\mathrm{T}, \hat{r}_{\mathrm{T}}}, \mathbf{R}_{x x}^{-1}\right) .
$$

Note that this contribution increases for increasing $\hat{r}_{\mathrm{S}}$ and $\hat{r}_{\mathrm{T}}$, while the distortion term $\mathrm{MSE}_{\mathrm{d}}$ decreases. An optimal tradeoff can be found by selecting the rank orders that minimize the MSE for the specific interference arrangement defined by the structure of the covariance $\mathbf{Q}$. For $\hat{r}_{\mathrm{S}}=M$ and $\hat{r}_{\mathrm{T}}=W$, the MSE reduces to the performance of the unconstrained channel estimate that is $\mathrm{MSE}_{\mathrm{u}}=\Phi\left(\mathbf{I}_{M}, \mathbf{Q}\right) \Phi\left(\mathbf{I}_{W}, \mathbf{R}_{x x}^{-1}\right)$.

\section{Antenna ArRay Configurations WITH DIRECTIVE ELEMENTS}

Assuming $M$ antenna elements, each with directivity gain $D(\theta)=\beta \cos ^{\gamma}(\theta)$, where $\theta$ is the direction of arrival (DOA), the following antenna array configurations are studied: A) The Uniform Circular Array (UCA) is shown in Fig. 2-(a) and the UCA vector $\mathbf{a}_{c}(\theta) \in \mathbb{C}^{M \times 1}$ in (2) is given by:

$$
\mathbf{a}_{c}(\theta)=\mathbf{D}_{c}(\theta) \circ \overline{\mathbf{a}}_{c}(\theta),
$$

where $\mathbf{D}_{c}(\theta) \in \mathbb{C}^{M \times 1}$ is the circular directivity vector and $\overline{\mathbf{a}}_{c}(\theta) \in \mathbb{C}^{M \times 1}$ is the array manifold, whose entries are 


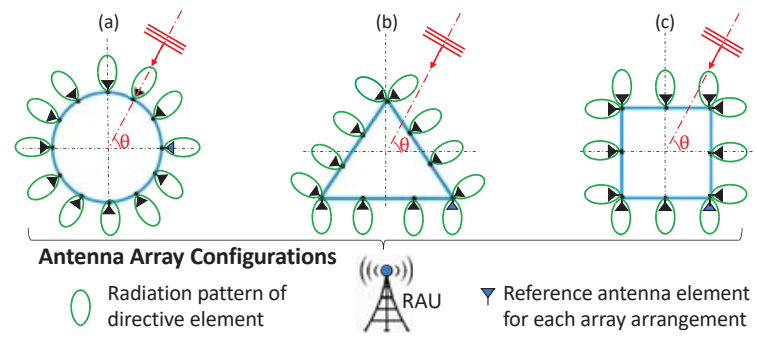

Figure 2. Different antenna arrays configurations at RAU: (a) circular, (b) triangular and (c) square arrangements.

respectively defined in the second and third column of the Table I, with $\lambda$ being the wavelength, $d=\frac{2 \pi r}{M}$ the interelement spacing, $r$ the array radius and $m=0, \ldots, M-1$. Moreover, o denotes the Hadamard (element-wise) product.

B) The Triangular Array (TA) is formed by three uniform linear arrays (ULAs) deployed over the three sides of a triangle (Fig. 2-(b)). The TA vector $\mathbf{a}_{t}(\theta) \in \mathbb{C}^{M \times 1}$ is:

$$
\mathbf{a}_{t}(\theta)=\mathbf{D}_{t}(\theta) \circ \overline{\mathbf{a}}_{t}(\theta)
$$

with $\mathbf{D}_{t}(\theta)=\left[D_{t_{1}}(\theta) D_{t_{2}}(\theta) D_{t_{3}}(\theta)\right]^{\mathrm{T}} \otimes \mathbf{1}_{M / 3} \in \mathbb{C}^{M \times 1}$ accounting for the three ULAs arrangement, with elements defined in the second column of Table I, with $\mathbf{1}_{m}$ being the vector with all entries equal to 1 . The array vector is $\overline{\mathbf{a}}_{t}(\theta)=$ $\left[\overline{\mathbf{a}}_{t_{1}}^{\mathrm{T}}(\theta) \overline{\mathbf{a}}_{t_{2}}^{\mathrm{T}}(\theta) \overline{\mathbf{a}}_{t_{3}}^{\mathrm{T}}(\theta)\right]^{\mathrm{T}}$, where the entry $m$ of each $\overline{\mathbf{a}}_{t_{i}}(\theta) \in$ $\mathbb{C}^{\frac{M}{3} \times 1}$, with $i=1, \ldots, 3$, is in the third column of Table I, with $\kappa=\frac{2 \pi}{\lambda}$ being the wavenumber and $m=0, \ldots, M / 3-1$.

C) The Square Array (SA) is formed by four ULAs deployed over the four sides of a square (Fig. 2-(c)). The SA vector $\mathbf{a}_{s}(\theta) \in \mathbb{C}^{M \times 1}$ is:

$$
\mathbf{a}_{s}(\theta)=\mathbf{D}_{s}(\theta) \circ \overline{\mathbf{a}}_{s}(\theta),
$$

with $\mathbf{D}_{s}(\theta)=\left[D_{s_{1}}(\theta) D_{s_{2}}(\theta) D_{s_{3}}(\theta) D_{s_{4}}(\theta)\right]^{\mathrm{T}} \otimes \mathbf{1}_{M / 4} \in \mathbb{C}^{M \times 1}$ based on the four ULAs arrangement, with directive elements given in the second column of Table I. The array manifold is $\overline{\mathbf{a}}_{s}(\theta)=\left[\overline{\mathbf{a}}_{s_{1}}^{\mathrm{T}}(\theta), \overline{\mathbf{a}}_{s_{2}}^{\mathrm{T}}(\theta), \overline{\mathbf{a}}_{s_{3}}^{\mathrm{T}}(\theta), \overline{\mathbf{a}}_{s_{4}}^{\mathrm{T}}(\theta)\right]^{\mathrm{T}}$, where the $m$ th element of each $\overline{\mathbf{a}}_{s_{i}}(\theta) \in \mathbb{C}^{M / 4 \times 1}$, with $i=1, \ldots, 4$, is defined in the third column of Table I, with $m=0, \ldots, M / 4-1$.

\section{Numerical ANALYSiS}

Performance of the proposed LR method is evaluated for varying rank orders and antenna array configurations, compared to the FR MLE. ST channel estimation is the first step in spatial precoding/decoding and temporal equalization, but the impact of the LR estimation onto spatial filtering capability, or any other processing, is outside the scope of the paper.

The mm-Wave uplink system operates with symbol rate $R_{s}=400 \mathrm{MHz}$ and carrier frequency $28 \mathrm{GHz}$. The distance between the user and the RAU is set to $R_{\mathrm{U}}=50 \mathrm{~m}$. The RAU is equipped with $M=48$ antennas with half-wavelength interelement spacing and directivity parameters set to $\beta=1.8$ and $\gamma=1.6$. The ST channel consists of $P=3$ paths, setting the sparsity degree and the rank orders, as exemplified in Fig. 3. The path amplitudes $\alpha_{p}(\ell), p=1,2,3$, are independent and identically distributed, with LOS-dominant Rician distribution,
Table I

\begin{tabular}{|c|c|c|}
\hline \multicolumn{2}{|c|}{ Shape Directivity $\mathbf{D}(\theta)$} & Array Manifold $\overline{\mathbf{a}}(\theta)$ \\
\hline$\bigcirc$ & $D_{c_{m}}(\theta)=D\left(\theta-\frac{2 \pi}{M} m\right)$ & $\bar{a}_{c_{m}}(\theta)=e^{j \frac{d M}{\lambda}\left(1-\cos \left(\theta=\frac{2 \pi}{M} m\right)\right)}$ \\
\hline \multirow{3}{*}{$\triangle$} & $D_{t_{1}}(\theta)=D\left(-\frac{\pi}{2}-\theta\right)$ & $\bar{a}_{t_{1 m}}(\theta)=e^{j \kappa d m \cos (\theta)}$ \\
\hline & $D_{t_{2}}(\theta)=D\left(\frac{5 \pi}{6}-\theta\right)$ & $\bar{a}_{t_{2_{m}}}(\theta)=e^{j \kappa d\left[\left(\frac{M}{3}-1\right) \cos (\theta)-m \cos \left(\frac{\pi}{3}-\theta\right)\right]}$ \\
\hline & $D_{t_{3}}(\theta)=D\left(\frac{\pi}{6}-\theta\right)$ & $\bar{a}_{t_{3 m}}(\theta)=e^{j \kappa d m \cos \left(\theta+\frac{\pi}{3}\right)}$ \\
\hline \multirow{4}{*}{$\square$} & $D_{s_{1}}(\theta)=D\left(-\frac{\pi}{2}-\theta\right)$ & $\bar{a}_{s_{1 m}}(\theta)=e^{j \kappa d m \cos (\theta)}$ \\
\hline & $D_{s_{2}}(\theta)=D(\pi-\theta)$ & $\bar{a}_{s_{2 m}}(\theta)=e^{j \kappa d\left[\left(\frac{M}{4}-1\right) \cos (\theta)-m \sin (\theta)\right]}$ \\
\hline & $D_{s_{3}}(\theta)=D\left(\frac{\pi}{2}-\theta\right)$ & $\bar{a}_{s_{3_{m}}}(\theta)=e^{j \kappa d\left[-\left(\frac{M}{4}-1\right) \sin (\theta)+m \cos (\theta)\right]}$ \\
\hline & $D_{s_{4}}(\theta)=D(\theta)$ & $\bar{a}_{s_{4}}(\theta)=e^{j \kappa d(-m \sin (\theta))}$ \\
\hline
\end{tabular}

ANTENNA ARRAY PARAMETERS.

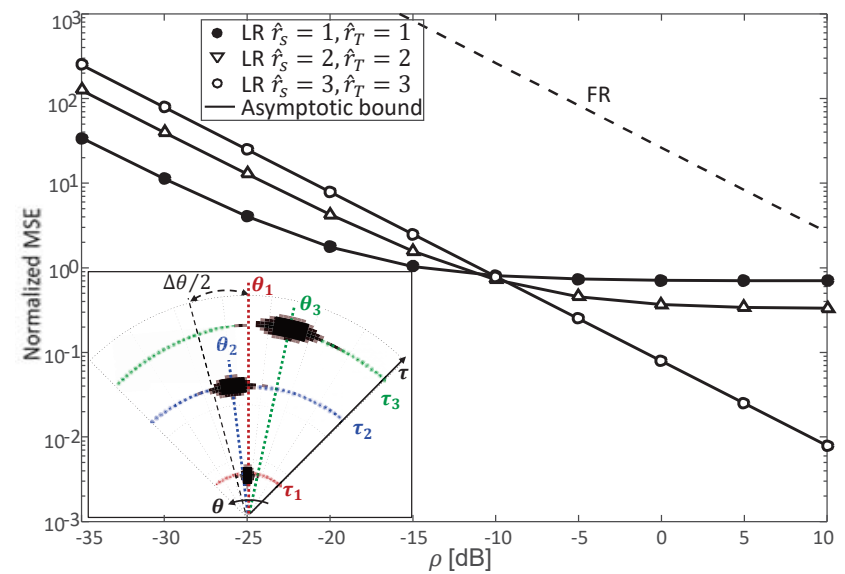

Figure 3. Normalized MSE vs omni-directional SIR $\rho$ for the FR and LR methods with $\hat{r}_{\mathrm{S}}=\hat{r}_{\mathrm{T}}=\{1,2,3\}$ for UCA arrangement at RAU and $\mathrm{SNR}=45 \mathrm{~dB}$. Subfigure: Example of ST propagation channel.

$\left|\alpha_{p}(\ell)\right| \sim \operatorname{Rice}\left(\sigma_{p}^{2}, K\right)$, with mean power $\sigma_{p}^{2}=\bar{P}_{r} / 3$ and $\mathrm{K}$-factor $K=10 \mathrm{~dB}$ according to the vertical-vertical (VV) antenna polarization model as typical mm-Wave small-scale fading [15]. $\bar{P}_{r}$ is the total received power computed assuming transmission power $20 \mathrm{dBm}$ and free-space path-loss. The angles of arrival of paths $p=2,3$ are uniformly distributed within an angular spread $\Delta \theta=\pi / 10 \mathrm{rad}$ around the DOA of the first path $\theta_{1}$. The delay of the first path is $\tau_{1}=R_{\mathrm{U}} / c$, being $c$ the vacuum velocity of light, while the delays of paths $p=2,3$ are in the range $\left[\tau_{1}, \tau_{m}\right]$, with $\tau_{m}=1.2 \tau_{1}=0.2 \mu \mathrm{s}$ computed based on the maximum angular spread $\Delta \theta$, with an overall delay spread of $\delta \tau=\tau_{m}-\tau_{1}=33.33 \mathrm{~ns}$. The pulse shaping $g(t)$ is a raised cosine with roll-off 0.2 and the temporal channel support is set to $W=40>R_{s} \delta \tau$. Moreover, the training sequence with $N=2 W$ quadrature phase shift keying (QPSK) symbols is simulated as a random sequence such that $\mathbb{E}\left[\mathbf{X X}^{\mathrm{H}}\right]=N \sigma_{x}^{2} \mathbf{I}_{W}$. The mm-Wave scenario includes $N_{I}=100$ interferers, operating at the same carrier frequency of the user and uniformly surrounding the RAU (as in Fig. 1), with DoAs $\theta_{I_{k}} \sim \mathcal{U}(0,2 \pi)$, with $k=1, \ldots, N_{I}$, and amplitudes modeled as Gaussian with covariance matrix $\mathbf{Q}_{\mathrm{I}}=\frac{\sigma_{I}^{2}}{N_{I}} \sum_{k=1}^{N_{I}} \mathbf{a}\left(\theta_{I_{k}}\right) \mathbf{a}^{\mathrm{H}}\left(\theta_{I_{k}}\right)$. The spatial covariance matrix is $\mathbf{Q}=\mathbf{Q}_{\mathrm{I}}+\mathbf{Q}_{\mathrm{N}}$, with $\mathbf{Q}_{\mathrm{N}}=\sigma_{\mathrm{N}}^{2} \mathbf{I}_{M}$ accounting for background noise. The signal-to-interference ratio (SIR) is defined as $\operatorname{SIR}=\sigma_{x}^{2} \mathbb{E}\left[\|\mathbf{H}(\ell)\|^{2}\right] /\left(M \sigma_{\mathrm{I}}^{2}\right)$ and the SNR as 

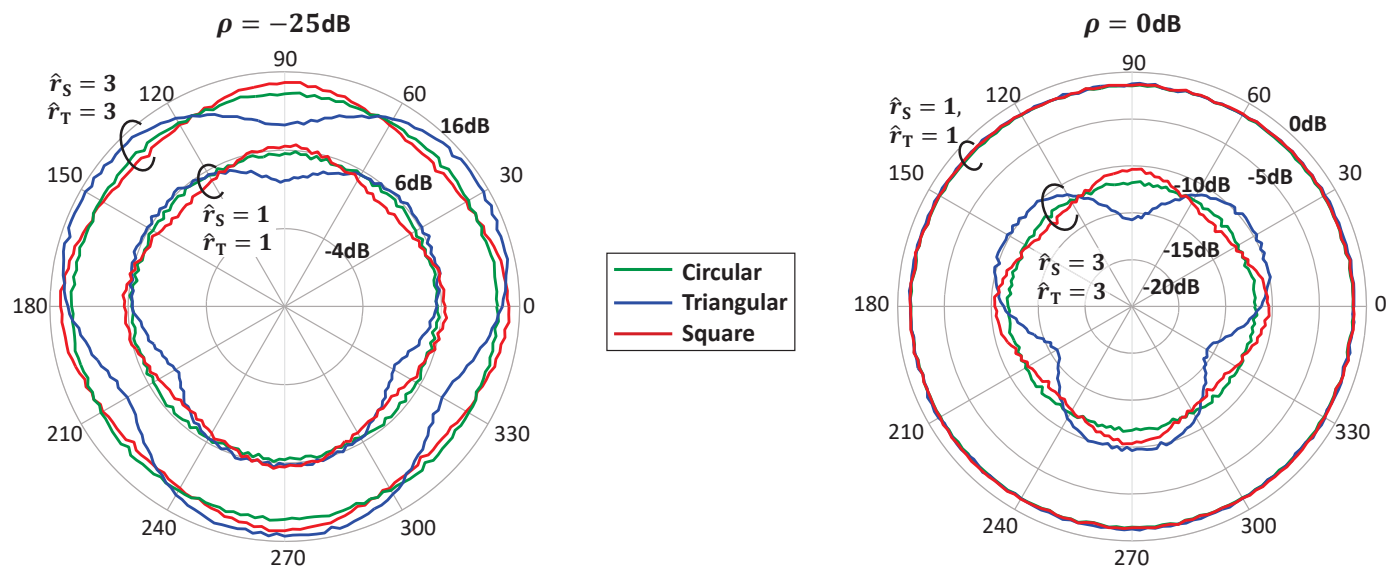

Figure 4. MSE angular analysis versus user direction $\theta_{U}=[0,2 \pi]$ for the LR method with $\hat{r}_{\mathrm{S}}=\{1,3\}$ and $\hat{r}_{\mathrm{T}}=\{1,3\}$ for circular (green), triangular (blue) and square (red) arrays and for $\rho=-25 \mathrm{~dB}$ (left) and $\rho=0 \mathrm{~dB}$ (right) and $\mathrm{SNR}=45 \mathrm{~dB}$.

$\mathrm{SNR}=\sigma_{x}^{2} \mathbb{E}\left[\|\mathbf{H}(\ell)\|^{2}\right] /\left(M \sigma_{\mathrm{N}}^{2}\right)$. Moreover, we define the SIR for omni-directional antennas at RAU as $\rho=\left(R_{\mathrm{I}} / R_{\mathrm{U}}\right)^{2}$, with $R_{\mathrm{I}}$ being the distance between one interferer and the RAU. This omni-directional SIR sets the geometrical parameters of the users/interferers deployment that purposely depends only on the relative mutual distances $R_{\mathrm{I}} / R_{\mathrm{U}}$. Note that for $N_{I}$ interferes, the experienced SIR for all interferers is $\rho / N_{I}$.

Fig. 3 shows the average performance of the proposed LR method and of the conventional FR solution, evaluated in terms of normalized MSE $=\mathbb{E}\left[\|\Delta \mathbf{H}(\ell) \mid\|^{2}\right] / \mathbb{E}\left[\|\mathbf{H}(\ell)\|^{2}\right]$ for varying omni-directional SIR $\rho$. Numerical averages are over 200 independent realizations of user-interferers random arrangements. The LR method is performed using $\hat{r}_{\mathrm{S}}=\hat{r}_{\mathrm{T}}=$ $\{1,2,3\}$ for UCA arrangement. Numerical results show that the performance of the LR estimator (markers) outperforms the FR MLE (dashed line) and closely attains the LR bound (solid line) evaluated as in (12). Moreover, for severe SIR conditions $(\rho<-10 \mathrm{~dB})$ rank-1 provides the best performance.

The angular analysis of the MSE bound versus the direction of the user $\theta_{U}=[0,2 \pi]$ is shown in Fig. 4 for different antenna arrays (circular, square and triangular shapes), for $\rho=\{-25,0\} \mathrm{dB}$. The MSE is in radial-units and confirms that for very-low SIR (left in Fig. 4 ) a rank-1 estimate $\left(\hat{r}_{\mathrm{S}}=1\right.$ and $\left.\hat{r}_{\mathrm{T}}=1\right)$ is beneficial compared to a rank- 3 estimate $\left(\hat{r}_{\mathrm{S}}=3\right.$ and $\hat{r}_{\mathrm{T}}=3$ ). On the other hand, when SIR is higher (right in Fig. 4), a rank-3 estimate is mandatory to attain the minimum MSE. When $\rho=-25 \mathrm{~dB}$ (left in Fig. 4) the performance degradation of the MSE for rank- 1 is about $6 \mathrm{~dB}$ compared to the $\rho=0 \mathrm{~dB}$ case (right in Fig. 4), supporting rank-1 channel estimation in sparse mm-Wave propagation settings.

\section{CONCLUSIONS}

In this paper, we investigated the feasibility of using the algebraic structure of sparse mm-Wave links to improve the performance of ST channel-interference estimation, for an uncalibrated array of antennas. Performance results show that the proposed LR method outperforms the conventional FR method. Moreover, even if true-rank is unknown, the rank-1 estimate is the preferred solution as it trades (mild) complexity with capability to operate in low SIR conditions. Numerical analysis shows that circular array is the preferable arrangement compared to the triangular and the square configurations.

\section{REFERENCES}

[1] T. S. Rappaport et al., "Millimeter Wave Mobile Communications for 5G Cellular: It Will Work!" IEEE Access, vol. 1, pp. 335-349, 2013.

[2] M. Z. Win et al., "A mathematical theory of network interference and its applications," Proc. of the IEEE, vol. 97, no. 2, pp. 205-230, Feb. 2009.

[3] A. Conti et al., "Log-concavity property of the error probability with application to local bounds for wireless communications," IEEE Trans. on Information Theory, vol. 55, no. 6, pp. 2766-2775, Jun. 2009.

[4] F. Rusek et al., "Scaling up MIMO: Opportunities and challenges with very large arrays," IEEE Signal Proc. Mag., vol. 30, no. 1, pp. 40-60, 2013.

[5] S. Han et al., "Large-scale antenna systems with hybrid analog and digital beamforming for millimeter wave 5G," IEEE Commun. Mag., vol. 53, no. 1, pp. 186-194, 2015.

[6] O. E. Ayach et al., "Spatially sparse precoding in millimeter wave MIMO systems," IEEE Trans. on Wireless Commun., vol. 13, no. 3, pp. 1499-1513, Mar. 2014.

[7] J. Gambini, "Precoding design with emission levels control for mmwave hybrid two-stage architectures," in IEEE ISWCS, Aug. 2017, pp. 152 157.

[8] R. W. Heath et al., "An overview of signal processing techniques for millimeter wave MIMO systems," IEEE J. of Sel. Topics in Signal Proc., vol. 10, no. 3, pp. 436-453, 2016.

[9] S. Sun and T. S. Rappaport, "Millimeter Wave MIMO channel estimation based on adaptive compressed sensing," in IEEE ICC Workshops, May 2017, pp. 47-53.

[10] Z. Gao et al., "Channel estimation for millimeter-wave massive MIMO with hybrid precoding over frequency-selective fading channels," IEEE Commun. Letters, vol. 20, no. 6, pp. 1259-1262, Jun. 2016.

[11] H. Ghauch et al., "Subspace estimation and decomposition for large millimeter-wave MIMO systems," IEEE J. of Sel. Topics in Signal Proc., vol. 10, no. 3, pp. 528-542, 2016.

[12] S. Haghighatshoar and G. Caire, "Massive mimo channel subspace estimation from low-dimensional projections," IEEE Trans. on Signal Proc., vol. 65, no. 2, pp. 303-318, Jan. 2017.

[13] S. Buzzi and C. D'Andrea, "Subspace tracking algorithms for millimeter wave mimo channel estimation with hybrid beamforming," in 21th International ITG WSA, Mar. 2017, pp. 1-6.

[14] M. Nicoli et al., "Multislot estimation of fast-varying space-time communication channels," IEEE Trans. on Signal Proc., vol. 51, no. 5, pp. 1184-1195, 2003.

[15] M. K. Samimi et al., "28 GHz millimeter-wave ultrawideband smallscale fading models in wireless channels," in IEEE 83rd VTC Spring, 2016, pp. 1-6. 